und Indiens, wobei Indien der Vorsitz zukommt. Laut Artikel 11 verpflichten sich sowohl Ägypten als auch die britische Kolonialmacht, unmittelbar nach der Anerkennung der Selbstbestimmung durch das sudanesische Parlament alle Truppen aus dem Sudan zurückzuziehen. Im Zuge einer maximal dreijährigen Transitionsphase soll die einzusetzende sudanesische verfassungsgebende Versammlung laut Artikel 9 über den Status des Sudan entscheiden: entweder eine Anbindung an Ägypten, oder vollständige Unabhängigkeit - ein Vertragsmodell, das frappant an das 2005 unterzeichnete CPA zwischen Sudan und der SPLA/M erinnert (Rolandsen und Daly, 2016: 66).

Die Frage des Südens streift der Vertrag nur an einer Stelle, ebenfalls in besagtem Artikel 9. Die verfassungsgebende Versammlung "will be to decide the future of the Sudan as one integral whole«. Weitere Bestimmungen zu einem etwaigen besonderen Status des Südens oder der Form seiner Eingliederung in einen unabhängigen Sudan finden sich nicht. Weder die sudanesische noch die ägyptische Seite zeigten daran Interesse. Dementsprechend trifft die Unabhängigkeit in der kleinen politischen Szene des Südens auf gemischte Gefühle, die unvermeidlich in enttäuschte Erwartungshaltungen umschlagen werden. »The cause was reluctantly supported by the South, which stipulated federalism and guarantees for the region as conditions for endorsing independence. The South opted for independence on the basis of Northern reassurances that their concerns would be given sserious consideration<. However, the North quickly reneged on promises to Southerners and stepped into the British colonial shoes.«(Deng, 2005: 6)

\title{
Wirkungen des Kolonialismus
}

Die Entwicklung des, je nach Sichtweise, südsudanesischen Widerstandsoder Befreiungskampfes ist eng mit dem durch das Kolonialregime verankerten binären Narrativ der angeblich »afrikanischen« und »arabischen« Identitäten der jeweiligen Regionen verknüpft. Natürlich beruht dieses Narrativ auf verschiedenen Erscheinungsformen. Es spiegelt die Phasen einer mitunter brutalen Eindringung ägyptischer Kolonialtruppen und arabischer Stämme wider. Ebenso beruht es auf der Wahrnehmung der später von wechselnden Zentralregierungen in Khartum brutal vorangetriebenen Sudanisierungspolitik. 
Dennoch liegt eine grundlegende Problematik in der geschichtlichen Erzählung eines kontinuierlichen Gewaltverhältnisses zwischen dem Norden und dem Süden (Rolandsen und Daly, 2016: 5; Mosely Lesch, 1998: 212-213; Deng, 1995: 492). Trotz verschiedener gewaltsam geprägter Konfliktlandschaften, neben dem politischen Projekt der Sudanisierung vor allem die Menschenjagden durch arabische Stämme (Jok, 2010), lässt sich die Erzählung als solche nicht aufrechterhalten. Sie impliziert ein Niveau an Vergesellschaftung, das zum Zeitpunkt der staatlichen Unabhängigkeit des Sudan weder im Norden noch im Süden erreicht war. Vielmehr beruht die Erzählung auf antikolonialen Vorstellungswelten auf beiden Seiten, dem Vorhalt einer versuchten Abspaltung des Südens einerseits, dem Vorhalt einer gezielten arabisch-islamischen Durchdringung andererseits. Die Southern Policy hat einen nachhaltigen Beitrag zur diskursiven Verankerung dieser Vorstellungen geleistet. Die nachhaltige Spaltung, zu der die Southern Policy innerhalb des Südens, vor allem zwischen Greater Bahr el-Ghazal und der Nil-Region, beigetragen hat, wird von dieser Sichtweise substanziell vernachlässigt.

Das letztlich abgerissene koloniale Projekt hat verschiedene einschneidende Konsequenzen für die Periode der sudanesischen Unabhängigkeit. Im Gegensatz zu benachbarten Kolonien kann der Süden des Sudan nicht auf eine einheitliche und verankerte Struktur politischer Institutionen zurückgreifen. Es muss davon ausgegangen werden, dass die Bevölkerung im Süden zu jenem Zeitpunkt in ihrem überwiegenden Teil kein Bewusstsein über Fragen wie Unabhängigkeit oder Staatlichkeit hat. »[A]s late as the eve of Sudan's independence in 1956, only at most an embryonic and elite sSouthern < political identity can be discerned.« (Rolandsen und Daly, 2016: 9)

Vielmehr werden die unterschiedlichen Gemeinschaften durch die versuchte Durchsetzung dieser Projekte in einen sie vereinheitlichenden Status der Marginalisierung und Entrechtung gedrängt, der zuvor unbekannt war.

»It must be emphasized that it is not mere differences that cause conflicts, but the implications for the shaping and sharing of power, wealth, services, development opportunities, and the overall enjoyment of the rights of citizenship. By this yardstick, the South clearly found itself at independence the most marginalized and discriminated region in the country.«(Deng, 2006: 157) 
